\title{
Ovarian Tissue Transplantation: Current State and Research Advancement
}

\author{
Chi-Huang Chen • Chii-Ruey Tzeng
}

Published online: 23 August 2013

(C) Springer Science+Business Media New York 2013

\begin{abstract}
During the past decade, babies born from cryopreservation and transplantation of ovarian tissue have emerged as a promising alternative to restore ovarian function and fertility in cancer patients. Despite successful results obtained with conventional freezing protocols to preserve ovarian tissue, attempt for whole ovary transplantation is still underpropelling in large animal and human studies. Patient selection to avoid reimplanting cancer cells in ovarian grafts as a prerequisite safety concern is as important as advanced transplantation technique. Multidisciplinary strategies for the combined isolation immature follicle for culture or in vitro activation of ovarian follicle with the frozen-thawed ovarian tissue raised the diversity for female fertility preservation along with the egg-embryo freezing or in vitro maturation of immature eggs. The purpose of this updated review of fertility preservation techniques is to develop a network among health care professional interested in this challenging field for those who are threatened the reproductive function.
\end{abstract}

Keywords Fertility preservation · Ovary · Orthotopic autotransplantation $\cdot$ Heterotopic autotransplantation cryopreservation $\cdot$ Female infertility

\section{Introduction}

Advances in cancer therapy have improved the long-term survival of cancer patients to make implementation of

C.-H. Chen · C.-R. Tzeng

Department of Obstetrics and Gynecology, School of Medicine,

College of Medicine, Taipei Medical University, Taipei, Taiwan

C.-H. Chen $\cdot$ C.-R. Tzeng $(\triangle)$

Department of Obstetrics and Gynecology, Center for Reproductive

Medicine, Taipei Medical University Hospital,

No. 252, Wuxing Street, Taipei 110, Taiwan

e-mail: d102095012@gmail.com procedures aimed at preserving fertility for young victims. These procedures must not be limited to preserve and restore fertility in patients undergoing therapies but also be applied to any cases where the reproductive function is threatened due to benign ovarian disease or primary ovarian insufficiency (POI).

Experimental and clinical strategies for preserving fertility include ovariostasis, embryo cryopreservation (IVF), cryopreservation of mature and immature oocytes, reimplantation of cryopreserved ovarian cortex, culture for ovarian tissue, preantral follicles and oocyte-cumulus complexes, whole ovary cryopreservation and transplantation, in vitro maturation of immature (IVM) oocytes [1•] (Table 1 ), and in vitro activation (IVA) of ovarian follicles [2•]. Among the currently available options for fertility preservation, restoration of ovarian activity and pregnancy after transplantation of cryopreserved ovarian tissue is rapidly gaining found as a valid method alongside cryopreservation of embryos and oocytes.

The best follicular survival rate obtained to date with frozen-thawed human ovarian tissue is approximately 70 $80 \%[3,4]$; primordial follicles are more resistant to cryoinjury. Earlier work revealed that almost $50 \%$ of primordial follicles are lost as a result of initial ischemia rather than as a result of the freeze-thaw process itself [5-7]. The majority of previous studies related to ovarian tissue cryopreservation and transplantation have focused on short-term viability [8]. Given the current accumulation of experience for ovarian cryopreservation, a slow cooling and rapid rewarming rate for mouse and human ovarian tissue $[9,10]$ or vitrification for sheep and monkey ovarian tissue effectively preserves primordial follicles [11, 12].

Tissue ischemia without vascular anastomosis remains a problem for implants because the process of revascularization can take 2-7 days to complete, dependent on factors including the size of implanted tissue [13, 14]. In theory, cryopreservation of intact ovary in vitro, followed by restoration of reproductive function after autologous transplantation using microvascular anastomosis, could achieve an immediate blood 
Table 1 Strategy of fertility preservation in women

Fertility preservation in women

\section{Ovariostasis}

Cryopreservation of mature and immature oocytes

Culture procedures for ovarian tissue, preantral follicles and oocytecumulus complexes

Progress in cryopreservation
Toxicity of chemotherapy and radiotherapy

Cryopreservation of ovarian cortex technical aspects

Cryopreservation of ovarian cortex Reimplantation of cryopreserved ovarian cortex: technique and results
Embryo cryopreservation (IVF)

Whole ovary cryopreservation

Whole ovary transplantation Lesson learned from experimental studies

Note: Adapted from http://www.isfp-fertility.org [1•]

supply in vivo to maximize graft survival. The success is subjected to improve freezing and implantation technique, graft function and lifespan, patient selection, identification of tumor involvement in the ovaries, and detection of small number of cancer cells in ovarian tissue.

To address these challenges, we aimed at the current state of basic and research regarding to transplantation of frozenthawed ovarian tissue, influence of the site of ovarian transplantation, environmental factor with possible effects on transplantation, patient criteria before transplantation, method and monitoring of transplanted grafts, safety issue after transplantation.

\section{Patient Selection}

Criteria for ovarian tissue banking (by S. Samuel Kim)

1) Age: younger than 37 years (may be individualized based on the status of ovarian reserve)

2) Ovarian function: premenopausal by FSH, antral follicle count (AFC), or AMH

3) Communication with oncologists: cancer treatment plan, prognosis

4) When embryo freezing or oocyte freezing is not indicated: delaying cancer treatment is not acceptable, hormonal stimulation is not permitted, ART is not allowed.

5) Prepubertal girls who do not have any other options

6) High risk for POF (when significant loss of ovarian follicles is anticipated with cancer therapy)

7) Informed consent from adult patients

8) Informed consent from parents/guardians as well as informed assent from minors, if the patient is younger than age 18 years

9) Physically and mentally healthy enough for surgery

10) Desires to have a child in the future (preferably before age 50 years)

11) Thorough patient counseling: currently available fertility preservation options, including embryo and oocyte cryopreservation, how to use cryobanked ovarian tissue for fertility restoration

12) Should understand experimental nature and potential risks of cancer cell transmission [15•].
Live Birth After Transplantation of Frozen-Thawed Ovarian Tissue

Transplantation of frozen-thawed ovarian tissue for fertility restoration and regaining of menstrual cycles is rapidly gaining ground as a valid method. A relative constant period elapses from transplantation until the tissue become active, also when a second transplant is performed. A total of 24 live births after orthotopic autotransplantation of slow-freezing technique have been reported between 2004 and 2013 [16•]. Only a few groups overwhelmed the majority of limited successes, including by Donnez's group in Belgium and Andersen's group in Denmark [16•].

Silber backs up the promising technique for a series of fresh ovarian isografts between genetically identical monozygotic twins to yield live birth [17]. Despite good evidence to support that the transplanted tissue is active, myth or reality for ovarian tissue transplantation remains a matter of concern. Progress in improving freezing techniques and enhance the avascular reimplantation to increase pregnancy rates or combined culture isolated immature oocytes is still challenging [16•].

Orthotopic autotransplantation of ovarian cortex has advantages, such as easy collection of ova and the possibility of spontaneous pregnancy. Although children have been born after successful orthotopic autotransplantation into the residual ovaries, some patients cannot undergo this procedure such as those who need bilateral ovariectomy of pelvic radiation therapy; therefore, it is still necessary to investigate suitable heterotopic autotransplantation sites in cynomolgus monkey [18]. A surgical technique for heterotopic transplantation of ovarian tissue to the forearm was reported by Oktay, of the eight oocytes suitable for in vitro fertilisation, one fertilized normally and developed into a four-cell embryo without embryo transfer [19, 20].

When ovarian tissue to a subcutaneous site and orthotopically to the pelvic peritoneum or to the ovaries, follicles developed more often in ovarian sites than at the peritoneal or subcutaneous sites. Furthermore, follicle development was limited at the heterotopic site $(<15 \mathrm{~mm})$ and oocyte recovery rate and fertilization rate obtained from heterotopic sites were low $[21,22]$. 
As high as $>50 \%$ of primordial follicle loss after reimplantation due to tissue ischemia after transplantation while awaiting angiogenesis. Donnez emphasized the importance of preparing the vascular bed to favor faster revascularization of the graft, because enhanced vascularization of the vascular bed is likely to increase vascularization in the grafted cortex [16•]. Ovarian cortex cryopreservation can be performed at the time of surgery for benign diseases when fertility is impaired. The first pregnancy to occur after ovarian tissue cryopreservation for benign ovarian pathology after bilateral oophorectomy was reported [23•].

\section{Monitor Ovarian Restoration}

Clinical workup before ovarian tissue harvesting also should include investigation of patient's medical status, investigation of possible risk of ovarian involvement, and consultation with an anesthesiologist. The main points for clinical evaluation of patients before ovarian tissue harvesting are presented in the list given below:

(1) Diagnosis, staging;

(2) Sterilization risk: disease, treatment protocol, age;

(3) Previous exposure to chemotherapy: time of treatment, protocol, menstrual history;

(4) Ovarian reserve:

(a) Hormones: gonadotrophins, E2, P, anti-Müllerian hormone (AMH);

(b) Sonography: total AFC ovarian volume;

(5) Evaluation of medical status;

(a) Leukemia: anemia, thrombocytopenia;

(b) Lymphoma: mass, pressure, effusion;

(c) Previous exposure to chemotherapy;

(ii) Anemia, thrombocytopenia;

(iii) Liver function;

(iiii) Renal function;

(iiv) Heart evaluation - echo;

(6) Risk of ovarian involvement sonography, computed tomography (CT) [24].

Ovarian function recovery was evaluated by hormone concentration measurement, follicular development on ultrasound, and menstruation recovery. There are individual variations in the duration of endocrine function after transplantation. The mean duration is $\sim 4-5$ years if follicular density is well preserved [16•].

The first signs of ovarian function restoration (oestradiol peak, decrease in FSH, ultrasound showing follicular development) occurred between 16 and 26 weeks after reimplantation [25]. There were three case reports describe the long-term duration of function of ovarian cortical tissue grafts among patients in a university fertility preservation programme in Europe and in a private practice programme in the United States. One woman underwent sterilizing cancer treatment and had frozen ovarian tissue transplanted, and two women underwent fresh ovarian tissue transplants. The function of ovarian cortical strips has continued for more than 7 years in these three women, with the birth of eight healthy babies following a single graft per patient [26 $]$.

Large numbers of primordial follicles were present in the harvested tissue. During the first months after transplantation, gonadotropins were high, AMH and inhibin B were low, and in three IVF cycles, eggs were not found. After recovery of endocrine activity 9 months after transplantation, a mature oocyte was retrieved. Embryo transfer resulted in a normal pregnancy and delivery of a healthy baby. Although spontaneous menstruation resumed after delivery, endocrine profile 22 months after transplantation indicated low reserve [27]. The recovery of endocrine function after transplantation correlated with the result of oocyte recovery. Fertility preservation using ovarian tissue is effective also in cases when the ovaries are injured after chemotherapy. However, transplant life span is limited [27]. Low AMH and inhibin B concentrations may suggest decreased ovarian reserve in women after ovarian transplantation. AMH and inhibin B levels may not be associated with the duration of ovarian graft function or probability to achieve a pregnancy [28•].

\section{Vitrification and Human Ovarian Tissue}

Vitrification procedures appeared to preserve not only the morphology and survival of preantral follicles after 1 week of xenografting, but also their ability to resume folliculogenesis. In addition, vitrification protocol had a positive impact on the quiescent state of primordial follicles after xenografting [29•]. Despite the encouraging results obtained with vitrification of ovarian tissue from humans and different animal species, it is necessary to understand how vitrification solutions and protocols can affect ovarian tissue, notably preantral follicles. In addition, it is important to bear in mind that the utilization of different approaches to assess tissue functionality and oocyte quality is essential in order to validate the promising results already obtained with vitrification procedures [30•]. However, so far there have been no human live births after ovarian vitrification.

\section{Whole Ovary Transplantation}

Spontaneous pregnancy after transplantation of frozenthawed rat upper genital tract and intact ovary with microvascular anastomosis shows that this technique has promise [31]. Subsequently, transplantation of whole fresh ovaries has been successfully performed in rats [31,32], rabbit [33], sheep [34, 35], monkey [36], and human [17, 37, 38], Leporrier et al. [39] and Hilders et al. [40], and Chen et al. reported that autologous 
heterotopic transplantation of intact rabbit ovary after frozen banking at $-196{ }^{\circ} \mathrm{C}$ [41]. Between 2003 and 2009, four groups reported whole ovary cryopreservation in sheep, and only one pregnancy was achieved [42]. There have been a total of four experiments of whole ovary cryopreservation without transplantation in humans [42]. Large animal studies have been encouraging, and it is likely that this option for fertility preservation will be a viable treatment option in the future.

Current research to determine the optimal technique for whole-ovary cryopreservation remains a challenge compared with tissue and cell cryopreservation; techniques are still in relative infancy. Combined microvascular manipulation with perfusion of cryoprotectant agents through intact organ vessels is a prerequisite for whole-ovarian cryopreservation and microvascular anastomosis. Long-term longevity and functional performance of whole cryopreserved ovary still is unclear [43]. If current obstacles to successful cryopreservation of ovarian tissue were overcome, transplantation could provide an abundant source of oocytes and hormone secretion appropriate for prolonged reproductive function. The choice of the recipient pedicle has to fulfill several criteria [44•].

- Easy surgical accessibility of the pedicle

- Feasible orthotopic transplantation into the pelvic cavity

- Optimal size match between the recipient pedicle and the gonadic vessels

- Possible postoperative ultrasound monitoring of the ovarian graft

- $\quad$ Easy puncture of the transplant for IVF

Given the significant improvement over the ischemic loss of follicles that is seen with cortical strip cryopreservation and retransplantation, cryopreservation of the whole ovary could potentially provide a superior alternative to patients requiring this method of fertility preservation. Large animal studies have been performed [45].

Ten monozygotic (MZ) twin pairs requested ovarian transplantation and nine have undergone transplantation with cryopreservation of spare tissue. Eight had a fresh cortical tissue transplant, one of whom received a second frozen-thawed transplant after the first ceased functioning at 3 years. One had a fresh microvascular transplant. All recipients reinitiated ovulatory menstrual cycles and normal day 3 serum folliclestimulating hormone levels by 77 to 142 days. Seven have already conceived naturally (three twice). Currently, seven healthy infants have been delivered out of ten pregnancies. The oldest transplant ceased functioning by 3 years, but then she conceived again after a frozen-thawed secondary transplant. There was no apparent difference in return of ovarian function between the nine fresh ovarian grafts and the one frozen graft. Ovarian transplantation appears to restore ovulatory function robustly. Ten pregnancies, leading to seven healthy infants, including one after cryopreservation, bode well for application to fertility preservation [46]. Fertility preservation, from cancer to benign disease to social reasons: the challenge of the present decade is proposed [47•].

Xenograft

Host and graft vessels contributed sequentially to graft revascularization: murine angiogenesis initiated reperfusion from day 5 , and by day 10 , human angiogenesis was shown to participate in graft revascularization. Host and graft angiogenesis are potential targets to reduce the avascular period after grafting [48]. The results demonstrate, for the first time, that isolated human follicles are able to survive and grow after xenografting. This study also shows massive in vivo follicular activation after transplantation of grafted fragments and isolated follicles. One week after grafting, well-structured stromalike tissue of human origin was observed around the isolated follicles [49]. The study demonstrates, for the first time, that cryopreservation and xenotransplantation do not appear to greatly affect human primordial/primary follicle ultrastructure.

Interestingly, in frozen-thawed xenografts, secondary human ovarian follicles presented a well-preserved ultrastructure, but asynchrony between oocyte and granulosa cell development was detected [50]. Impact of freezing and thawing of human ovarian tissue on follicular growth after long-term xenotransplantation is nevertheless warranted to confirm the identity of theca cells and assess if they retain the ability to respond to luteinizing hormone and produce androgens [51•].

Iso- and Allografts

Ovarian transplantation between monozygotic twins was discordant for premature ovarian failure [52]. Allotransplantation might be a remedy. Silber and colleagues reported a series of monozygotic twins who underwent ovarian isotransplantation to rescue the sterile sister $[46,52,53]$.

Furthermore, orthotopic ovarian allotransplantation has been performed on patients diagnosed with Turner syndrome. This restored regular menstruation and ovulation raised hormonal concentrations and led to the development of secondary sexual characters $[38,54]$. This manuscript reports the first live birth after allografting between monozygotic twins with 45,XO/46,XX mosaicism and discordant growth pattern and ovarian function [55•].

The present state of transplant biology, as both a basic and a clinical science, is unlikely to have been achieved without lessons learned from rodent transplant models. Progress in this area has occurred in several ways during the past few decades, including organ preservation, overcoming ischaemia/reperfusion injury and immune rejection, and the development and testing of new immunosuppressive regimens. Many efforts have been made to evaluate possible rejection and survival models of solid organ transplantation and to generate monitoring systems for tissues, such as the pancreatic islets, kidney, 
liver, and heart [56-59]. However, few studies have addressed the pathophysiology of ovarian allografts, which are indispensable in the development of ovarian transplantation [60].

In an in vivo rodent model to evaluate the rejection of ovarian allografts while mice were treated with or without cocktail immunosuppressant [61], luciferin bioluminescence imaging (BLI) was used to follow graft survival, and viable follicle numbers were counted as a measure of success. Bioluminescence intensity fluctuated but was consistent with the endpoint counts of viable follicle numbers. Graft survival and measurable bioluminescence for up to 30 days in NOD-SCID mice and allografts treated with immunosuppressive therapy. Without immunosuppressant, the ovarian grafts were rejected completely as early as 10 days following ovarian engraftment.

On the contrary, even in the absence of immunosuppressive therapy, ovarian allografting between genetically nonidentical sisters allowed restoration of ovarian function in cases where previous bone marrow transplantation from the HLAcompatible sister resulted in full chimerism, avoiding the threat of rejection in a case report [62].

In human model, ten $\mathrm{MZ}$ twin pairs requested ovarian transplantation and eight have undergone transplantation with cryopreservation of spare tissue. Seven had a fresh cortical tissue transplant, one of whom received a second frozenthawed transplant after the first ceased functioning at 3 years. One had a fresh microvascular transplant. All recipients reinitiated ovulatory menstrual cycles and normal Day 3 serum FSH levels by 77-142 days. Six have already conceived naturally (one twice). Currently, two healthy babies have been delivered, and another three pregnancies are ongoing. The oldest transplant functioned for 36 months, resulting in one child and one miscarriage [17].

\section{In Vitro Activation of Ovarian Follicles}

In patients with primary ovarian insufficiency, also known as premature ovarian failure (POF), early exhaustion of ovarian follicles is characterized by high serum FSH along with amenorrhea before 40 years of age. Earlier studies demonstrated that oocyte-specific deletion of the phosphatase and tensin homolog (PTEN) gene led to the activation of dormant primordial follicles. After IVA of primordial follicles by incubating ovaries from neonatal mice with PTEN inhibitors and a phosphatidylinositide 3-kinase PI3K stimulating peptide, ovaries were transplanted under kidney capsules of ovariectomized hosts. Primordial follicles in ovarian grafts developed to the preovulatory stage containing mature eggs. After in vitro fertilization and embryo transfer, healthy progeny were delivered [63].

Kawamura applied PTEN inhibitor and PI3K stimulators to activate dormant primordial follicles in vitro, following by xenografting of human cortical fragments into immunedeficient mice, mature human oocytes were generated from dormant primordial follicles after 6 months. Based on the success to obtain human oocytes by the IVA procedure, this application for POI patients for fertility preservation turns out to be possible after ovarian grafts transplanted in the mesosalpinx to yield mature oocytes, followed by in vitro fertilization and embryo transfer [2•].

Facilitate Angiogenesis and Protect Avascular Ovarian Grafts

In contrast to cryopreservation, which seems to have only a small deleterious effect on the primordial follicles, there is some evidence that the key factor responsible for follicle loss is postgrafting ischemia with hypoxia. This ischemia causes a proportion of cortical cells to undergo apoptosis after grafting. The ovary is a three-dimensional structure composed of gametes and somatic cells. During the transplantation process, complete revascularization takes time, and the transplantation site selected is important. Therefore, reducing the damage caused by reperfusion-ischemia is an issue requiring urgent resolution. Yang et al. demonstrated that the majority of apoptotic processes occur within the first week after transplantation, before well-organized blood vessels are established between the graft and the host [64].

The survival of transplanted human ovarian tissue may be improved by treatment of both the host (with vitamin E and gonadotropins) and the graft (with vascular endothelial growth factor A [VEGF-A] and vitamins) [65•]. Five young patients with cancer donated ovarian tissues that were transplanted into immunodeficient mice. Graft survival improved after the host was treated with melatonin, or the graft was incubated with hemagglutinin-rich biological glue, especially when combined with VEGF-A and vitamin E [66•]. Tsai et al. [67•] reported that the protective effects of the antiapoptotic agent sphingosine-1-phosphate (S1P) on vitrified ovarian grafts to prove the S1P protects vitrified ovarian grafts from ischemic reperfusion injury rather than from vitrification-associated process.

Live birth in a woman without ovaries after autograft of frozen-thawed ovarian tissue combined with growth factors plasma rich in platelets (PRP) to promote neoangiogenesis [68•].

Coadministration of an immunomodulator, trichloro (dioxoethylene-O,O') tellurate (AS101), reduced follicle activation, thereby increasing follicle reserve and rescuing fertility after cyclophosphamide (Cy), and also increased the efficacy of Cy against breast cancer cell lines. These findings suggest that the mechanism in Cy-induced loss of ovarian reserve is accelerated primordial follicle activation, which results in a "burnout" effect and follicle depletion. By preventing this activation, AS101 shows potential as an ovarian-protective agent, which may be able to preserve fertility in female cancer patients [69•].

Chorioallantoic membrane (CAM) model provides a new approach to study human ovarian tissue transplantation in its 


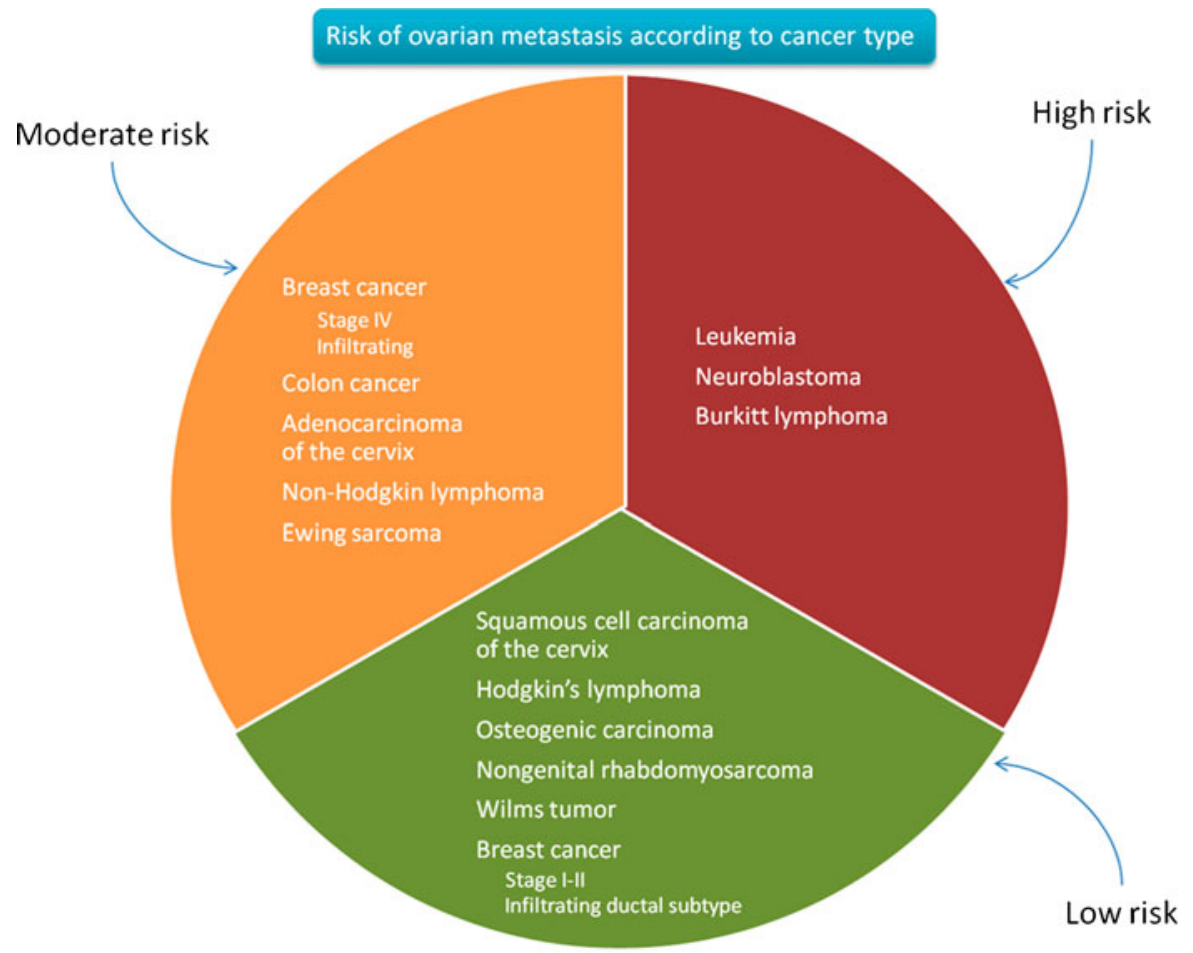

Note: Adapted from Dolmans MM et al. 2013 [77]

Fig. 1 Risk of ovarian metastasis according to cancer type

first ischemic stages, yielding information on the timing of tissue changes before the establishment of neovascularization [70].

Among the large series of 60 patients who underwent reimplantation of cryopreserved ovarian tissue, 11 conceived and 6 of those had already delivered 12 healthy babies. Donnez pointed out in the future to look to: 1) improve freezing techniques; and 2) enhance the "vascular bed" before reimplantation to increase pregnancy rates [16•]. Cryopreservation of ovarian tissue may be combined with removal, via puncture, of small antral follicles, making it possible to freeze both ovarian tissue and isolated immature oocytes for in vitro maturation [16•].

An alginate-based matrix is a promising proposition to graft isolated ovarian cells. After transplantation, this matrix was able to degrade, allowed vascularization and elicited a low inflammatory response $[71 \bullet]$.

\section{Safety Issue}

Pregnancy and live birth after autotransplantation of frozenthawed ovarian tissue in a patient with metastatic disease underwent chemotherapy and hematopoietic stem cell transplantation [72]. By quantitative RT-PCR, two of six CML patients were positive for $B C R-A B L$ in their ovarian tissue. Among the 12 ALL patients, 7 of the 10 with available molecular markers showed positive leukemic markers in their ovarian tissue (translocations or rearrangement genes). Four mice grafted with ovarian tissue from ALL patients developed intraperitoneal leukemic masses. This study demonstrates, by quantitative RT-PCR, ovarian contamination by malignant cells in acute as well as chronic leukemia, whereas histology fails to do so. Moreover, chemotherapy before ovarian cryopreservation does not exclude malignant contamination. Finally, reimplantation of cryopreserved ovarian tissue from ALL and CML patients puts them at risk of disease recurrence [73, 74•] (Fig. 1).

\section{Conclusions}

Fertility preservation by the cryopreservation of ovarian tissue is a new but increasingly successful clinical option. The extraction of ovarian tissue can be performed easily by laparoscopy, and the risk of transplantation of cancer cells remains very low in a number of diseases. This technique therefore should be considered in women in the mid-thirties or younger receiving highly gonadotoxic chemotherapy or pelvic radiation. The cryopreservation of ovarian tissue requires the establishment of centralised and highly specialist cryobanks as well as sophisticated networks to integrate oncologists and reproductive specialists in order to provide this procedure to all patients in need [75]. 
Acknowledgments This work was supported by NSC99-2314-B-038033-MY3 from the National Science Council, Taiwan, R.O.C.

\section{Compliance with Ethics Guidelines}

Conflict of Interest Chi-Huang Chen and Chii-Ruey Tzeng declare that they have no conflict of interest.

Human and Animal Rights and Informed Consent This article does not contain any studies with human or animal subjects performed by any of the authors.

\section{References}

Papers of particular interest, published recently, have been highlighted as:

- Of importance

1. International society for fertility preservation: fertility preservation in women. http://www.isfp-fertility.org/members-only/scientificarticles/fertility-preservation -in-women/. Accessed July 2013. It is cited from the web of "International Society for Fertility Preservation "(ISFP)" to guide the strategy of fertility preservation in women.

2. - Kazuhiro Kawamura: Treatment of POI patients based on in vitro activation (IVA) of ovarian follicles [lecture, page: 32]. Presented at The International Ovarian Conference. Taipei, Taiwan; March 9-10, 2013. The lecture of invited speaker, Kazuhiro Kawamura, who piloted a cutting edge application for those POI patients by in vitro activation of primordial follicle of ovarian tissue, followed by frozen, transplantation, ovarian stimulation, egg retrieval, and embryo transfer in humans.

3. Newton H, Aubard Y, Rutherford A, et al. Low temperature storage and grafting of human ovarian tissue. Hum Reprod. 1996;11:1487-91.

4. Gook DA, Edgar DH, Stern C. Effect of cooling rate and dehydration regimen on the histological appearance of human ovarian cortex following cryopreservation in 1, 2-propanediol. Hum Reprod. 1999;14:2061-8.

5. Nugent $D$, Neirow $D$, Brook PF, et al. Transplantation in reproductive medicine: previous experience, present knowledge and future prospects. Hum Reprod Update. 1997;3:267-80.

6. Baird DT, Webb R, Campbell BK, et al. Long-term ovarian function in sheep after ovariectomy and transplantation of autografts stored at $-196^{\circ}$. Endocrinology. 1999;140:462-71.

7. Gosden RG. Low temperature storage and grafting of human ovarian tissue. Mol Cell Endocrinol. 2000;163:125-9.

8. Torrents E, Boiso I, Barri PN. Applications of ovarian tissue transplantation in experimental biology and medicine. Hum Reprod Update. 2003;9:471-81.

9. Cox SL, Shaw J, Jenkin G. Transplantation of cryopreserved fetal ovarian tissue to adult recipients in mice. J Reprod Fertil. 1996;107:315-22.

10. Gook DA, Edgar DH, Stern C. The effects of cryopreservation regimens on the morphology of human ovarian tissue. Mol Cell Endocrinol. 2000;169:99-103.

11. Courbiere B, Massardier J, Salle B, et al. Follicular viability and histological assessment after cryopreservation of whole sheep ovaries with vascular pedicle by vitrification. Fertil Steril. 2005;84:1065-71.

12. Yeoman RR, Wolf DP, Lee DM. Coculture of monkey ovarian tissue increases survival after vitrification and slow-rate freezing. Fertil Steril. 2005;83:1248-54.
13. Dissen GA, Lara HE, Fahrenbach WH, et al. Immature rat ovaries become revascularized rapidly after autotransplantation and show a gonadotropin -dependent increase in angiogenic factor gene expression. Endocrinology. 1994;134:1146-54.

14. Gosden RG, Baird DT, Wade JC, Webb R. Restoration of fertility to oophorectomized sheep by ovarian autografts stored at $-196{ }^{\circ} \mathrm{C}$. Hum Reprod. 1994;9:597-603.

15. • ISFP Practice Committee, Kim SS, Donnez J, Barri P, et al. Recommendations for fertility preservation in patients with lymphoma, leukemia, and breast cancer. J Assist Reprod Genet. 2012;29:465-8. Recommendations for fertility preservation in patients with lymphoma, leukemia, and breast cancer. The committee has pioneered the frozen-thawed ovarian tissue in young cancer patient or those who are threatened by ovarian failure.

16. • Donnez J, Dolmans MM, Pellicer A, et al. Restoration of ovarian activity and pregnancy after transplantation of cryopreserved ovarian tissue: a review of 60 cases of reimplantation. Fertil Steril. 2013;99:1503-13. Donnez is the first and most experienced master to yield the majority of reported live birth after frozen-thawed ovarian tissue in young cancer patients. This article is the largest series study to review 60 cases of restoration of ovarian activity and pregnancy after transplantation of cryopreserved ovarian tissue.

17. Silber SJ, DeRosa M, Pineda J, et al. A series of monozygotic twins discordant for ovarian failure: ovary transplantation (cortical versus microvascular) and cryopreservation. Hum Reprod. 2008;23:1531-7.

18. Nao Suzuki, Shu Hashimoto, Suguru Igarashi, et al.: Heterotopic autotransplantation of ovarian cortex in cynomolgus monkeys [OC17]. Presented at World Congress on Fertility Preservation. Brussels, Belgium; December 10-12, 2009.

19. Oktay K, Buyuk E, Rosenwaks Z, Rucinski J. A technique for transplantation of ovarian cortical strips to the forearm. Fertil Steril. 2003;80:193-8.

20. Oktay K, Buyuk E, Veeck L, et al. Embryo development after heterotopic transplantation of cryopreserved ovarian tissue. Lancet. 2004;363:837-40.

21. Demeestere I, Simon P, Buxant F, et al. Ovarian function and spontaneous pregnancy after combined heterotopic and orthotopic cryopreserved ovarian tissue transplantation in a patient previously treated with bone marrow transplantation: case report. Hum Reprod. 2006;21:2010-4.

22. Demeestere I, Simon P, Emiliani S, et al. Orthotopic and heterotopic ovarian tissue transplantation. Hum Reprod Update. 2009;15:649-65.

23. - Donnez J, Jadoul P, Pirard C, et al. Live birth after transplantation of frozen-thawed ovarian tissue after bilateral oophorectomy for benign disease. Fertil Steril. 2012;98:720-5. Donnez's group reports the experience for live birth after transplantation of frozen-thawed ovarian tissue after bilateral oophorectomy for benign disease.

24. Meirow D. Fertility preservation in cancer patients using stored ovarian tissue: clinical aspects. Curr Opin Endocrinol Diabetes Obes. 2008; $15: 536-47$.

25. Donnez J, Squifflet J, Van Eyck AS, et al. Restoration of ovarian function in orthotopically transplanted cryopreserved ovarian tissue: a pilot experience. Reprod Biomed Online. 2008;16:694-704.

26. • Andersen CY, Silber SJ, Bergholdt SH, et al. Long-term duration of function of ovarian tissue transplants: case reports. Reprod Biomed Online. 2012;25:128-32. This article reported the longest lifespan of ovarian tissue transplants from the Andersen's group in Denmark, which also is famed for success of live birth after frozen-thawed ovarian tissue in young cancer patients.

27. Meirow D, Levron J, Eldar-Geva T, et al. Monitoring the ovaries after autotransplantation of cryopreserved ovarian tissue: endocrine studies, in vitro fertilization cycles, and live birth. Fertil Steril. 2007;87:418.e7-418.e15.

28. - Janse F, Donnez J, Anckaert E, et al. Limited value of ovarian function markers following orthotopic transplantation of ovarian tissue after gonadotoxic treatment. J Clin Endocrinol Metab. 
2011;96:1136-44. Many serum markers have been applied to evaluate the functional restoration of frozen-thawed ovarian tissue. This article was published by JCEM to point out the limited value of ovarian function markers following orthotopic transplantation of ovarian tissue after gonadotoxic treatment.

29. - Amorim CA, Dolmans MM, David A, et al. Vitrification and xenografting of human ovarian tissue. Fertil Steril. 2012;98:12918.e1-2. Despite no human live birth after vitrified ovarian tissue, this technique has been paid much more attention and studies in recent years. Both articles are cited from Donne's group to discuss the advantages of vitrification protocol for ovarian tissue cryopreservation in humans and animal species and also resume primodial folliculogenesis after xenografting.

30. - Amorim CA, Curaba M, Van Langendonckt A, et al. Vitrification as an alternative means of cryopreserving ovarian tissue. Reprod Biomed Online. 2011;23:160-86. See previous annotation.

31. Wang X, Chen H, Yin H, et al. Fertility after intact ovary transplantation. Nature. 2002;415:385-6.

32. Yin H, Wang X, Kim SS, et al. Transplantation of intact rat gonads using vascular anastomosis: effects of cryopreservation, ischaemia and genotype. Hum Reprod. 2003;18:1165-72.

33. Winston RM, Browne JC. Pregnancy following autograft transplantation of Fallopian tube and ovary in the rabbit. Lancet. 1974;2:494-5.

34. Goding JR, McCracken JA, Baird DT. The study of ovarian function in the ewe by means of a vascular autotransplantation technique. $\mathrm{J}$ Endocrinol. 1967;39:37-52.

35. Jeremias E, Bedaiwy MA, Gurunluoglu R, et al. Heterotopic autotransplantation of the ovary with microvascular anastomosis: a novel surgical technique. Fertil Steril. 2002;77:1278-82.

36. Scott JR, Keye WR, Poulson AM, Reynolds WA. Microsurgical ovarian transplantation in the primate. Fertil Steril. 1981;36:512-5.

37. Martinez-Madrid B, Dolmans MM, Van Langendonckt A, et al. Freeze-thawing intact human ovary with its vascular pedicle with a passive cooling device. Fertil Steril. 2004;82:1390-4.

38. Mhatre P, Mhatre J, Magotra R. Ovarian transplant: a new frontier. Transplant Proc. 2005;37:1396-8.

39. Leporrier M, von Theobald P, Roffe JL, Muller G. A new technique to protect ovarian function before pelvic irradiation. Heterotopic ovarian autotransplantation. Cancer. 1987;60:2201-4.

40. Hilders CG, Baranski AG, Peters L, et al. Successful human ovarian autotransplantation to the upper arm. Cancer. 2004;101:2771-8.

41. Chen $\mathrm{CH}$, Chen SG, Wu GJ, et al. Autologous heterotopic transplantation of intact rabbit ovary after frozen banking at -196 degrees $\mathrm{C}$. Fertil Steril. 2006;86:1059-66.

42. Chian RC, Quinn P: Fertility cryopreservation. Edited by Chian RC, Quinn P. New York: Cambridge University Press; 236-238.

43. Bedaiwy MA, Jeremias E, Gurunluoglu R, et al. Restoration of ovarian function after autotransplantation of intact frozen-thawed sheep ovaries with microvascular anastomosis. Fertil Steril. 2003;79:594-602.

44. - Ploteau S, Rogez JM, Donnez J, Lengelé B. Which are the ideal donor and recipient vessels for a whole ovarian transplantation? Fertil Steril. 2011;95:751-5. Although frozen-thawed whole ovarian transplantation is debatable for its success, this article is worth leaning for the technique of ideal donor and recipient vessels for a whole ovarian transplantation.

45. Bromer JG, Patrizio P. Fertility preservation: the rationale for cryopreservation of the whole ovary. Semin Reprod Med. 2009;27:465-71.

46. Silber SJ. Fresh ovarian tissue and whole ovary transplantation. Semin Reprod Med. 2009;27:479-85.

47. - Donnez J. Introduction: fertility preservation, from cancer to benign disease to social reasons: the challenge of the present decade. Fertil Steril. 2013;99:1467-8. As a pioneer, Donnez intends to address that predicting the likelihood of infertility following gonadotoxic treatments is extremely difficult. In the present issue, several hot topics in the field of fertility preservation are discussed.
48. Van Eyck AS, Bouzin C, Feron O, et al. Both host and graft vessels contribute to revascularization of xenografted human ovarian tissue in a murine model. Fertil Steril. 2010;93:1676-85.

49. Dolmans MM, Martinez-Madrid B, Gadisseux E, et al. Short-term transplantation of isolated human ovarian follicles and cortical tissue into nude mice. Reproduction. 2007;134:253-62.

50. Nottola SA, Camboni A, Van Langendonckt A, et al. Cryopreservation and xenotransplantation of human ovarian tissue: an ultrastructural study. Fertil Steril. 2008;90:23-32.

51. - Amorim CA, David A, Dolmans MM, et al. Impact of freezing and thawing of human ovarian tissue on follicular growth after long-term xenotransplantation. J Assist Reprod Genet. 2011;28:1157-65. Antral follicles obtained after grafting of frozen-thawed human ovarian tissue showed a thinner theca cell layer compared with those from fresh grafts, which could affect follicular development and function. Further studies are nevertheless warranted to confirm the identity of theca cells and assess if they retain the ability to respond to luteinizing hormone and produce androgens.

52. Silber SJ, Lenahan KM, Levine DJ, et al. Ovarian transplantation between monozygotic twins discordant for premature ovarian failure. N Engl J Med. 2005;353:58-63.

53. Silber SJ, Grudzinskas G, Gosden RG. Successful pregnancy after microsurgical transplantation of an intact ovary. N Engl J Med. 2008;359:2617-8.

54. Mhatre P, Mhatre J. Orthotopic ovarian transplant-review and three surgical techniques. Pediatr Transplant. 2006;10:782-7.

55. - Donnez J, Dolmans MM, Squifflet J, et al. Live birth after allografting of ovarian cortex between monozygotic twins with Turner syndrome (45,XO/46,XX mosaicism) and discordant ovarian function. Fertil Steril. 2011;96:1407-11. Girls with Turner syndrome suffer accelerated loss of ovarian follicles and only 2-5\% experience spontaneous menses with the potential to achieve pregnancy without medical intervention. This manuscript reports the first live birth after allografting between monozygotic twins with 45,XO/46,XX mosaicism and discordant growth pattern and ovarian function .

56. Berman A, Pawelec K, Fiedor P. Allogeneic transplantation of isolated islet cells in clinical practice. Pol Arch Med Wewn. 2009;119:326-32.

57. Martínez-Dolz L, Almenar L, Reganon E, et al. What is the best biomarker for diagnosis of rejection in heart transplantation? Clin Transplant. 2009;23:672-80.

58. Shapiro AM, Lakey JR, Ryan EA, et al. Islet transplantation in seven patients with type 1 diabetes mellitus using a glucocorticoid-free immunosuppressive regimen. N Engl J Med. 2000;343:230-8.

59. Truong DQ, Bourdeaux C, Wieërs G, et al. The immunological monitoring of kidney and liver transplants in adult and pediatric recipients. Transpl Immunol. 2009;22:18-27.

60. Chen $\mathrm{CH}$, Yeh YC, Wu GJ, et al. Tracking the rejection and survival of mouse ovarian iso- and allografts in vivo with bioluminescent imaging. Reproduction. 2010;140:105-12.

61. Lin YH, Yeh YC, Tzeng CR, et al. Evaluating the effects of immunosuppression by in-vivo bioluminescence imaging after allotransplantation of ovarian grafts. Reprod Biomed Online. 2011;22:220-7.

62. Donnez J, Squifflet J, Pirard C, et al. Restoration of ovarian function after allografting of ovarian cortex between genetically non-identical sisters. Hum Reprod. 2010;25:2489-95.

63. Li J, Kawamura K, Cheng Y, et al. Activation of dormant ovarian follicles to generate mature eggs. Proc Natl Acad Sci U S A. 2010;107:10280-4.

64. Yang H, Lee HH, Lee HC, et al. Assessment of vascular endothelial growth factor expression and apoptosis in the ovarian graft: can exogenous gonadotropin promote angiogenesis after ovarian transplantation? Fertil Steril. 2008;90:1550-158.

65. - Abir R, Fisch B, Jessel S, et al. Improving posttransplantation survival of human ovarian tissue by treating the host and graft. Fertil Steril. 2011;95:1205-10. Improving posttransplantation survival of 
human ovarian tissue by treating the host and graft by vit-E, VEGF$A$, and gonadotropins.

66. • Friedman O, Orvieto R, Fisch B, et al. Possible improvements in human ovarian grafting by various host and graft treatments. Hum Reprod. 2012;27:474-82. The findings suggest that host treatment with melatonin or graft incubation with HA-rich biological glue, especially when combined with VEGF-A and vitamin $E$ improves graft survival. This protocol can be applied and holds promise in ovarian autotransplantation for fertility restoration.

67. - Tsai YC, Tzeng CR, Wang CW, et al.: Antiapoptotic agent sphingosine-1 -phosphate protects vitrified murine ovarian grafts. Reprod Sci 2013, in press. Rarely has antiapoptotic agent been applied in frozen-thawed transplanted ovarian tissue. This article reported that the antiapoptotic agent, Sphingosine-1-Phosphate $(S 1 P)$, protects vitrified ovarian grafts from ischemic reperfusion injury rather than from vitrification-associated process.

68. - Callejo J, Salvador C, González-Nuñez S, et al. Live birth in a woman without ovaries after autograft of frozen-thawed ovarian tissue combined with growth factors. J Ovarian Res. 2013;6:33. The authors reported a special interesting case, because this is the first time that this technique is performed successfully in a woman without ovaries combined with growth factors to promote neoangiogenesis. Obvious$l y$, the results of the hormonal response come exclusively from the implanted tissue in these special conditions.

69. - Kalich-Philosoph L, Roness H, Carmely A, et al. Cyclophosphamide triggers follicle activation and "burnout"; AS101 prevents follicle loss and preserves fertility. Sci Transl Med. 2013;5:185ra62. This article suggests that the mechanism in Cy-induced loss of ovarian reserve is accelerated primordial follicle activation, which results in a "burnout" effect and follicle depletion. By preventing this activation, AS101 shows potential as an ovarian-protective agent, which may be able to preserve fertility in female cancer patients.
70. Martinez-Madrid B, Donnez J, Van Eyck AS, et al. Chick embryo chorioallantoic membrane (CAM) model: a useful tool to study shortterm transplantation of cryopreserved human ovarian tissue. Fertil Steril. 2009;91:285-92.

71. - Vanacker J, Luyckx V, Dolmans MM, et al. Transplantation of an alginate-matrigel matrix containing isolated ovarian cells: first step in developing a biodegradable scaffold to transplant isolated preantral follicles and ovarian cells. Biomaterials. 2012;33:6079-85. By tissue engineering, these results demonstrate that an alginate-based matrix is a promising proposition to graft isolated OCs. After transplantation, this matrix was able to degrade, allowed vascularization, and elicited a low inflammatory response.

72. Donnez J, Squifflet J, Jadoul P, et al. Pregnancy and live birth after autotransplantation of frozen-thawed ovarian tissue in a patient with metastatic disease undergoing chemotherapy and hematopoietic stem cell transplantation. Fertil Steril. 2011;95:1787.e1-4.

73. Dolmans MM, Marinescu C, Saussoy P, et al. Reimplantation of cryopreserved ovarian tissue from patients with acute lymphoblastic leukemia is potentially unsafe. Blood. 2010;116:2908-14.

74. - Dolmans MM, Luyckx V, Donnez J, et al. Risk of transferring malignant cells with transplanted frozen-thawed ovarian tissue. Fertil Steril. 2013;99:1514-22. A review of the existing literature was done to evaluate the risk of transplanting malignant cells in case of the main malignant indications for ovarian tissue cryopreservation. For ovarian tissue from patients with hematologic malignancies, it is of paramount importance to identify minimal residual disease before ovarian tissue transplantation. Indeed, these pathologies, reviewed here in detail, are considered to be most at risk of ovarian metastasis .

75. von Wolff M, Donnez J, Hovatta O, et al. Cryopreservation and autotransplantation of human ovarian tissue prior to cytotoxic therapy-a technique in its infancy but already successful in fertility preservation. Eur J Cancer. 2009;45:1547-53. 